\title{
EDITORIAL
}

\section{Management of Diabetes Mellitus: Shifting Paradigm}

Though Diabetes Mellitus was known to mankind since dawn of civilization, until $1500 \mathrm{AD}$, medical science was mixture of clinical observation and philosophy. After 1500 AD experimental science started. Scientist observed sweet taste of diabetic urine, presence of sugar in urine, sweet taste of diabetic blood serum, charges in pancreas in diabetic subject, calcification of pancreas etc.

Paul Langerhans (1848-88) demonstrated the special tissue in pancreas i.e. Islets of Langerhans. E.L. Opie (1873-1921) observed reduced number of Islet in Diabetes. Landmark experimental study was done by Oscar Minkowski (1859-1931) and Joseph Von Mering (1849-1908) of Germany. They did pancreatectomy of a dog and that caused it diabetic, presence of ketone in urine. Then they injected the pancreatic tissue extract in the body of pancreatectomised dog and that proved to prevent diabetic symptoms. Though in 1907, William Lane distinguished A and B islet cells, beta cell first drew the attention.

\section{Discovery of Insulin}

Working at a University of Toronto Laboratory in 1921 Fred Banting and Charles Best were able to make a pancreatic extract which had antidiabetic characteristics. They were successful in testing their extract on diabetic dogs. Within months professor J.J.R MacLeod, who provided the lab space and general scientific direction to Banting and Best, put his entire research team to work on the production and purification of insulin. J.B. Collip joined the team and with his technical expertise the four discovers were able to purify insulin for use on diabetic patients. The first tests were conducted on Leonard Thompson early in 1922. These were a spectacular success. Word of this success spread quickly around the giving immediate hope to many diabetic persons who were near to death. A frenzied quest for insulin followed. Some patients in a diabetic coma made miraculous recoveries.

\section{Evolution of Insulins}

Animal Insulin

Beginning in 1922, and in the face of great demand for the new medicine, several companies were granted licenses by the University of Toronto to manufacture insulin. Initially patient needed several injections daily to maintain blood sugar level which was painful. In 1936, protamine, a low-weight protein, was used to develop a slow-release insulin.

In 1950 yet another approach led to the presently available isophane NPH (Neutral Protamine Hagedorn) insulin, which is also bound to protamine. It has a maximal effect of $24 \mathrm{~h}$ and can be mixed with any proportion of fast-acting regular insulin, which reduced injection number.

Despite the discovery of insulin scientist were in the quest of development oral medicine. In 1956, the first antidiabetic oral drugs - sulfonamide (tolbutamide, carbutamide) and biguanide derivatives (metormin, phenformin) - came to the market. But till today scientist are yet to develop ideal oral drug.

In 1974, chromatographic purification techniques allowed the production of highly purified animal insulin (less than $1 \mathrm{pmol} / \mathrm{l}$ of protein impurities). This product was called 'monocomponent MC'. Before this development, porcine and bovine insulin at times caused antibody allergies and lipoatrophy.

Human Insulin and Analogues

After several years of laboratory work, during the years 1963-1966 human insulin was chemically synthesized in Germany by Meienhofer et al. and in the United States by Katsoyannis et al. In 1978, scientists in San Francisco using a genetically manipulated plasmid of E. coli bacteria, succeeded in producing insulin with the same amino sequence as seen in humans.

Problems with conventional insulins: The need for analogues

Until some time back there are only human soluble shortacting (regular) insulin, intermediate-acting insulins 
(NPH and lente insulin) and mixtures of regular plus NPH insulin in various proportions (30/70 mixture being used more widely). The pharmacokinetics of these conventional insulin preparations fail to match the physiological insulin secretion profile, and due to this in most patients it has been virtually impossible to obtain $\mathrm{HbA}_{1} \mathrm{c}$ values around or $<7.0 \%{ }^{1,2}$. Attempts to reach the target of $\mathrm{HbA}_{1} \mathrm{c}<7 \%$, patients are facing hyperglycemia as well as hypoglycemia in post-meal state. Another drawback with these insulins are that a considerable number of patients have been characterized by a degree of control with a huge day-to-day variation in glycaemic level ${ }^{2}$. This variation is a result of several factors including variability in insulin action, insulin absorption, etc. and is observed both within an individual (intra individual) and between different individuals (inter individuals). Even though insulin therapy has evolved over the years with advances in purity, species, retarding agents, and other excipients, scientists were unable to overcome these inherent limitations of conventional insulins.

Search for newer insulins: meeting the unmet needs Efforts were focused at searching for better insulin formulations that can mimic endogenous insulin secretion more closely, so that optimal glycaemic control becomes a reality. It was the advent of biotechnology in the 1980s, which facilitated the efforts and helped scientists modify the native insulin molecule and design insulins with more desirable properties. The pioneering work on rapid-acting insulin analogues were performed by Brange and colleagues in the mid-80s $\mathrm{s}^{3,4}$. A later work on long-acting designer insuins was initiated. Several strategies were studied and a few of them were applied to bring out designer insulins, commonly called insulin analogues.

The concept of designer insulins has raised great expectations. The arrival of the rapid-acting analogues contributes to improved patients' convenience and reduced postprandial glucose excursions ${ }^{5}$ with a lower risk of hypoglycaemia. On the other hand the basal insulin analogues have fulfilled to a great extent the need for a more physiological basal insulin.

The availability of better monitoring tools along with the evidence to show that near-normal glycaemic control reduces the risk of diabetic complications has increased the need for insulin preparation that offer greater effectiveness, safety and versatility ${ }^{6}$. Insulin analogues have met this need to a great extend as these newer insulins have made possible the near-physiological replacement of prandial as well as basal insulin. The proper use of insulin analogues allows people with diabetes greater flexibility in the timing of meals, snacks, and exercise, which in turn enhances their ability to lead normal lives ${ }^{7}$. Their availability will help treatment strategies to be tailored to the needs of individual patients thereby helping them to achieve the best possible metabolic control. Insulin analogues will also provide physicians with the appropriate tools to overcome the obstacles to improve metabolic control and subsequently improve diabetes outcomes ${ }^{8,9}$.

\section{Glucagon Factor and Peptide Analogues}

If we consider pathophysiology of DM, we know it is not only insulin factor which is decreased and insulin resistance which prevent insulin to work efficiently are responsible, but there is also glucagons factor. In response to a carbohydrate meal, in nondiabetic subject not only there is increase is increase in insulin secretion but also simultaneously decrease in glucagon secretion. In contrast glucagons secretion in type 2 diabetics is not decreased, and may paradoxically increase. These produce an excessive postprandial glucose excursion by increased hepatic output.

Increatin hormones which are secreted in various parts of the gut e.g. glucagons-like peptide-1 (GLP-1) and glucose-dependent insulinotropic polypeptide (GIP), regulate glucose homeostasis by increasing insulin synthesis, decreasing glucagons secretion from pancreatic alpha-cells resulting in decreased hepatic out put it also works by slowing gastric empting and thus suppressing food intake. Both GLP-1 and GIP are rapidly inactivated by the enzyme dipeptidyl peptidase-4).

\section{Glucagon-like peptide (GLP) and analogs and agonists}

GLP agonists bind to a membrane GLP receptor. As a consequence of this, insulin release from the pancreatic beta-cells is increased. Endogenous GLP has a half life of only a few minutes; thus an analogue of GLP was produced.

- Exenatide is the first GLP-1 agonist approved for the treatment of type 2 diabetes. It is not an analogue of GLP, but rather a GLP agonist and has only 53\% homology with GLP, which increases its resistance 
to degradation by DPP-4 and extends its half-life. Typical reductions in A1C values are $0.5-1.0 \%{ }^{10}$.

- Liraglutide, a once daily human analogue (97\% homology), is rather better GLP-1 analogue. Animal model shows that GLP-1 analogue preserve even regenerate $\mathrm{B}$-cell mass.

These agents may also cause a decrease in gastric motility, responsible for the common side effect of nausea, and is probably the mechanism by which weight loss occurs.

\section{DPP-4 inhibitors:}

Dipeptidyl peptidase-4 (DPP-4) inhibitors increase blood concentration of the incretin GLP-1 (Glucagonlike peptide-1) by inhibiting its degradation by dipeptidyl peptidase-4 (DPP-4). They are vildagliptin, sitagliptin, saxagliptin etc. But there are also limitations in their action.

\section{Conclusion:}

Management of diabetes actually started after the discovery of insulin. From insulin scientist tried to shift to oral drug. But observing the limitations of oral drugs both secretogogues and senstizer, attention focused again of insulin with various modification of its formulation. Simultaneously, keeping pathophysiology of diabetes, role of alpha cell secreting glucagons and various gut hormones are also now strongly focused. Another hormone secreted by B-cell i.e. Amylin is perhaps going to be another tool in managing diabetes mellitus.

Despite of development of newer molecules having capability of good glycemic status with less adverse effect, along with preservation and regeneration of Bcell mass, a good number of patients are going to have diabetes related complications. Modern treatment prolonging life expectancy of good number of patients with all deadly complications causing them a handicapped.
So, can we prevent development of DM? The answer is 'yes'. Prevention of DM does not cost any money rather it saves money. Many studies proved beyond doubt that simple life style modification by changing eating habits and increase in physical activity, DM can be prevented in $60-65 \%$ of subjects. So, there remains a great hope.

(J Bangladesh Coll Phys Surg 2012; 30: 123-125)

\section{Prof. Zafar Ahmed Latif}

Professor of Endocrinology

BIRDEM Hospital, Dhaka

\section{References:}

1. The Diabetes Control and Complications Trial (DCCT) Research Group. The effect of intensive treatment of diabetes on the development and progression ofd long term complications in insulin dependent diabetes mellitus. N Engl J Med 1993; 329: 977-86.

2. Binder C, Lauritzen T, Faber O, Pramming S. Insulin pharmacokinetics. Diabetes Care 1984; 7: 188-99.

3. Brange J, Ribel U, Hansen J. Monomeric insulins obtained by protein engineering and their medical implications. Nature 1988; 333: 679-82.

4. Barnett AH. A review of basal insulins. Diabet Med 2003; 20: 873-85.

5. Barnett AH, Owens DR. Insulin analogues. Lancet 1997; 349: 47-51.

6. Hirsch IB. Insulin analogues. N Engl J Med 2005; 352: $174-$ 83.

7. UK Prospective Diabetes Group (UKPDS) 13: Relative efficacy of randomly allocated diet, sulfonylurea, insulin, or metformin in patients with newly diagnosed non insulin dependent diabetes followed for three years. BMJ 1995; 310: 83-88.

8. Heinemann L. Variability of insulin absorption and insulin action. Diabetes Technol Ther 2002; 4(5): 673-82.

9. Heinemann L. Overcoming obstacles: new management options. Eur J Endocrinol 2004; 151: T23-T27.

10. Briones, Bajaj M. "Exenatide: a GLP-1 receptor agonist as novel therapy for type 2 diabetes mellitus”. Expert Opinion Pharmacotherapy 2006; 7(8): 1055-64. 